\title{
Cardiac Surgery at the Time of Simultaneous Liver-Kidney Transplantation
}

\author{
James M. Tatum ${ }^{\mathrm{a}, \mathrm{b}}$, Adrienne M. Quinn ${ }^{\mathrm{a}}$, Yuri S. Genyk ${ }^{\mathrm{a}}$, Linda S. Sher ${ }^{\mathrm{a}}$, Lea K. Matsuoka ${ }^{\mathrm{a}}$, \\ Amy E. Hackman ${ }^{\mathrm{a}}$, Sophoclis P. Alexopoulos ${ }^{\mathrm{a}}$, Mark L. Barra, Michael E. Bowdish ${ }^{\mathrm{a}}$, \\ Mark J. Cunningham ${ }^{\mathrm{a}}$
}

\begin{abstract}
Cardiac valve disease is often a contraindication to liver or simultaneous liver-kidney transplantation. The risk of heart surgery in these patients is extremely high, yet uncorrected valve disease jeopardizes transplantation outcomes. Two cases of combined aortic valve replacement at the time of simultaneous liver-kidney transplantation, with 2-month and 3-year postoperative follow-ups, are discussed. Both patients survived surgery and were discharged with good allograft and heart function. At last follow-up, neither experienced graft failure or valve dysfunction, although one patient did develop type 1B rejection of his kidney allograft. In appropriate cases, surgical correction of structural heart disease can be performed at the time of complex abdominal organ transplantation, accomplishing both otherwise contraindicated procedures.
\end{abstract}

Keywords: Simultaneous liver-kidney transplant; Cardiac surgery; Liver transplantation

\section{Introduction}

End-stage liver disease (ESLD) complicates cardiac surgery due to coagulopathy, poor nutritional status, immune dysfunction, cirrhotic cardiomyopathy and occasionally coexisting pulmonary and renal dysfunction [1]. Conversely, orthotopic liver transplantation (OLT) confers stressors such as fluid shifts, hemodynamic instability, electrolyte imbalances during liver reperfusion and postoperative immunosuppressive agents that potentially impact cardiac function. Valve disease can result in elevated right heart and central venous pressures caus-

Manuscript accepted for publication March 25, 2016

aDepartment of Surgery, Keck School of Medicine of the University of Southern California, 1520 San Pablo Street, Healthcare Consultation Center II, Suite 4300, Los Angeles, CA 90033, USA

${ }^{\mathrm{b}}$ Corresponding Author: James M. Tatum, Department of Surgery, Keck School of Medicine of the University of Southern California, 1520 San Pablo Street, Health Care Consultation Center II, Suite 4300, Los Angeles, CA 90033, USA. Email: James.Tatum@med.usc.edu

doi: http://dx.doi.org/10.14740/jcs300w ing venous congestion in abdominal organs. Left heart or aortic valve disease decreases forward flow to grafted abdominal organs resulting in decreased arterial perfusion. It is critical to assess the cardiac state of patients being listed for transplant and when lesions are identified, to decide if they can be addressed before abdominal transplant surgery. Both patients described in this report had insufficiency of the aortic valve secondary to resolved bacterial endocarditis. The incidence of bacterial endocarditis in patients awaiting transplantation is not noted in the literature. However, it is known that patients with cirrhosis have a relative risk of being diagnosed with endocarditis approximately double that of the general population [2].

Renal failure in ESLD patients, whether due to primary renal dysfunction or secondary to hepatorenal syndrome, complicates surgical and perioperative care. Simultaneous liverkidney transplantation (SLK) is performed in 6-7\% of patients undergoing liver transplantation as a means to improve outcomes in patients with hepatorenal syndrome secondary to the liver disease or in patients with independent chronic or acute kidney failure qualifying for renal transplantation $[3,4]$. In recent years, reports of combined OLT and non-transplant open cardiac surgery, including coronary artery bypass and valve replacement or repair, have been growing in number $[5,6]$.

Discussed here are two patients who underwent surgical repair of the aortic valve after medically treated endocarditis along with SLK as a single operation.

\section{Case Reports}

After Institutional Review Board approval, a retrospective review of two patients undergoing aortic valve replacement combined with SLK between 2013 and 2015 at the University of Southern California was performed.

Patients underwent extensive preoperative cardiac workup, including echocardiography, coronary angiography, and cardiac catheterization. Both patients were found to have severe aortic insufficiency secondary to aortic valve leaflet pathology.

\section{Case 1}

Case 1 is a 58 -year-old male with hepatitis $\mathrm{C}$ cirrhosis, a model for end-stage liver disease (MELD) score of 40 and non-oligu- 
ric acute kidney injury from diuretic use and type 1 hepatorenal syndrome. Serum creatinine was $3.10 \mathrm{mg} / \mathrm{dL}$ on hospital day 1. Continuous renal replacement therapy (CRRT) was initiated on the third day of hospitalization for volume overload. He required CRRT or hemodialysis (HD) until the day of operation and during surgery. Hospitalization began 21 days prior to transplant, and 19 of 21 days were in the intensive care unit.

Two months prior to admission, he had been diagnosed with group B Streptococcal bacteremia and completed a 1-week course of antibiotics at an outside medical center which were continued on transfer. During his preoperative cardiac evaluation, echocardiography as well as right and left heart catheterizations were performed revealing aortic valve vegetation presumed to be from endocarditis, causing severe aortic regurgitation with an ejection fraction (EF) of $62 \%$ and a mean pulmonary artery pressure of $14 \mathrm{~mm} \mathrm{Hg}$ with a cardiac output of $5.77 \mathrm{~L} / \mathrm{min}$. Multiple blood culture samples were negative preoperatively. The patient had a history of prior percutaneous coronary intervention with three stents in place and no significant coronary lesions on preoperative coronary catheterization. Mild tricuspid and mitral valve regurgitation were also identified.

After identification of a liver and kidney donor, the patient was taken to the operating room where he underwent open aortic valve replacement performed through a median sternotomy on cardiopulmonary bypass (CBP) with 30,000 units of heparin and cooling to a low of $32{ }^{\circ} \mathrm{C}$. A $23 \mathrm{~mm}$ tissue valve was used. After weaning $\mathrm{CPB}$, protamine administration and re-warming the chest was packed open and liver transplant was performed in piggy back fashion with caval preservation. This was followed by the kidney transplantation into the pelvis. Cold preservation times for the donor liver and kidney were 7.0 and 9.3 $\mathrm{h}$, respectively. The case concluded with chest closure.

Pathology revealed aseptic vegetations on the aortic valve leaflets. He completed a 6-week course of empiric ceftriaxone postoperatively.

Immunosuppression was initiated with a single dose of basiliximab in the operating room and a second dose on postoperative day (POD) 5, and was maintained with mycophenolate mofetil and tacrolimus. Immunosuppressive regimens were not modified due to cardiac surgery.

Postoperatively, case 1 had immediate return of liver function, with bile being produced by the grafted liver in the operating room. Liver function tests and international normalized ratio (INR) normalized early in his postoperative course. At 2-month follow-up, liver function tests and synthetic function were within normal limits.

Intermittent HD was continued until POD 25 when adequate renal function was achieved. Renal biopsy on POD 22 showed acute tubular necrosis without signs of rejection. Serum creatinine was $1.79 \mathrm{mg} / \mathrm{dL}$ at discharge and was $1.14 \mathrm{mg} /$ $\mathrm{dL}$ at 1-month follow-up. At 2-month follow-up, serum creatinine has increased to $2.04 \mathrm{mg} / \mathrm{dL}$, and biopsy of the renal allograft revealed type $1 \mathrm{~B}$ rejection.

Early postoperative hypotension required vasopressors to maintain adequate perfusion pressures. These were weaned off by POD 17 and he was transitioned to oral midodrine. Atrial fibrillation occurred on POD 16 and was treated with amiodarone. $\mathrm{EF}$ at this time was $40 \%$ with good overall cardiac func- tion. There were no signs of heart failure at any time during the postoperative period. He was weaned from oral midodrine on POD 38. At 1-month follow-up, oral amiodarone was discontinued with no return of arrhythmias at latest follow-up 2 months post-discharge. Left ventricular function was maintained at an EF of $40 \%$ with persistent mild global hypokinesia; prosthetic valve function was excellent with no leakage or regurgitation.

\section{Case 2}

Case 2 was a 55-year-old male with a history of ESLD secondary chronic rejection of a hepatic allograft performed 7 months prior to admission (initial transplant secondary to alcoholic cirrhosis). Patient was admitted from home for transplant with a MELD score of 38. Two months prior the acute-on-chronic renal failure developed secondary to hepatorenal syndrome and calcineurin inhibitor toxicity with a serum creatinine of $3.04 \mathrm{mg} / \mathrm{dL}$. Renal replacement therapy was initiated and he received $\mathrm{HD}$ as an outpatient while awaiting transplant.

Preoperative echocardiography revealed new severe aortic insufficiency with a mobile vegetation and perforation of the non-coronary cusp of his aortic valve. Cardiac function was normal with an EF of $62 \%$, and mild tricuspid regurgitation with a peak pulmonary artery systolic pressure estimated to be $51 \mathrm{~mm} \mathrm{Hg}$ by echocardiography. Left heart catheterization demonstrated normal coronary arteries. He was treated with broad spectrum antibiotics until multiple blood cultures returned negative and was continued on empiric ceftriaxone for 6 weeks prior to surgery.

On the day of surgery, he underwent open aortic valve replacement through a median sternotomy on CPB with 35,000 units of heparin and cooling to a low of $31^{\circ} \mathrm{C}$. A $25 \mathrm{~mm}$ porcine tissue aortic valve was used. Protamine was administered, the patient was re-warmed and CBP was weaned. The chest was left open as the liver and kidney transplants were performed. The liver re-transplantation was performed in a piggy back technique with caval preservation. The donor kidney was then transplanted in the pelvis. The chest was temporarily closed because of postoperative coagulopathy, and was formally closed the following day in the operating room. Pathologic evaluation revealed severe degeneration of the native aortic valve with an aseptic vegetation.

Liver function was regained in the operating room with bile being produced upon reperfusion. Liver function tests normalized prior to discharge on POD 16 and remained within normal limits at 2-year follow-up. Immunosuppression was maintained with tacrolimus, prednisone, and mycophenolate mofetil, and no changes to the usual protocol were made because of cardiac surgery.

At 2-year follow-up, he continued to have a functioning kidney allograft with a serum creatinine of $1.95 \mathrm{mg} / \mathrm{dL}$, never requiring dialysis after leaving the operating room.

Cardiac function was normal during the postoperative course except for an episode of atrial fibrillation treated with a 3-week course of oral amiodarone. He remained in sinus rhythm with excellent functional status since surgery without indication for repeat echocardiography or signs of cardiac dys- 
function.

\section{Discussion}

SLK has become common; however, it remains a procedure with some controversy and debatable indications [4]. Patients with irreversible kidney disease that will not improve after liver transplant alone do not clearly benefit from receiving a combined transplant procedure rather than liver transplant alone or liver transplant followed by continued renal replacement therapy and secondary listing for later renal transplant if indicated. Nonetheless, it is a procedure that is being performed and its appropriate indications are a subject of ongoing research.

Performing major operative procedures on patients with advanced liver disease risks high rates of morbidity and mortality. A recent review suggests cardiac surgery can be performed in carefully managed patients with Childs-Turcot-Pugh class A and possibly B liver disease or MELD score between 13 and 18 , but in Childs-Turcot-Pugh C patients, mortality after cardiac surgery is as high as $68 \%$ [1]. The feasibility of performing liver transplantation simultaneously with open cardiac surgery has been demonstrated [5-7]. A recent series from the Cleveland Clinic describes 10 patients classified as Childs-TurcotPugh B or C with a mean MELD score of 17 over an 11-year period who underwent cardiac procedures and simultaneous orthotopic liver transplantation, reporting a $20 \%$ perioperative mortality rate and $70 \%$ survival at 3 years; however, none of these patients had a simultaneous kidney transplant [6].

Multiple reports and reviews note the high perioperative mortality of performing cardiac surgery on patients with endstage renal disease, with early mortality rates as high as $7 \%$ [8]. The spectrum of cardiac disease in patients with both liver failure and renal failure requiring cardiac surgery is however almost certainly different given the high incidence of hepatorenal syndrome as an indication for dialysis in the pre-liver transplant population. Open cardiac surgery performed simultaneously with renal transplantation has been demonstrated to be as safe as temporally separate cardiac surgery and renal transplantation in patients with indications for both, with shorter times to extubation, hospital stays, and intensive care unit stays in part because of greater ease in attaining stable postoperative fluid and electrolyte balance in the postoperative period with a functional kidney [9].

We know the parts of this combination all work separately. With these cases, we report favorable short and medium term outcomes for two patients with dialysis dependent chronic renal failure, Childs-Turcot-Pugh C liver disease, MELD scores of $38-40$ and severe aortic insufficiency. Notably, both of these patients had preserved cardiac function with normal EFs preoperatively as well as normal or treated coronary arteries and isolated non-degenerative, non-ischemic valve disease. Both patients survived surgery and have had excellent outcomes. Limitations to this review are attributable to the medium or very short-term follow-up currently available and the small sample size. These cases support the idea that cardiac surgery at the time of SLK transplantation may be a reasonable option and an effective use of resources and may allow patients who are too sick to undergo liver and kidney transplant or cardiac surgery independent from each other to successfully undergo both.

\section{Conflicts of Interest}

The authors declare no conflict of interest.

\section{Author Contributions}

JMT and AMQ: manuscript preparation and data collection. YSG, LKM, AH, and SPA: manuscript review. LSS: manuscript preparation and conceptual planning. MLB, MEB, and MJC: manuscript preparation and review.

\section{Funding}

Division of Cardiothoracic Surgery Academic Research Funding.

\section{Abbreviations}

CABG: coronary artery bypass grafting; CPB: cardiopulmonary bypass; CRRT: continuous renal replacement therapy; EF: ejection fraction; ESLD: end-stage liver disease; HD: hemodialysis; INR: international normalized ratio; MELD: model for end-stage liver disease; OLT: orthotopic liver transplantation; POD: postoperative day; SLK: simultaneous liverkidney transplant

\section{References}

1. Lopez-Delgado JC, Esteve F, Javierre C, Ventura JL, Manez R, Farrero E, Torrado H, et al. Influence of cirrhosis in cardiac surgery outcomes. World J Hepatol. 2015;7(5):753-760.

2. Hung TH, Hsieh YH, Tseng KC, Tsai CC. The risk for bacterial endocarditis in cirrhotic patients: a population-based 3-year follow-up study. Int J Infect Dis. 2013;17(6):e391-393.

3. Brennan TV, Lunsford KE, Vagefi PA, Bostrom A, Ma M, Feng S. Renal outcomes of simultaneous liver-kidney transplantation compared to liver transplant alone for candidates with renal dysfunction. Clin Transplant. 2015;29(1):34-43.

4. Nadim MK, Davis CL, Sung R, Kellum JA, Genyk YS. Simultaneous liver-kidney transplantation: a survey of US transplant centers. Am J Transplant. 2012;12(11):31193127.

5. Davari HR, Malek-Hosseini SA, Salahi H, Bahador A, Nikeghbalian S, Nemati MH, Sanjarian MA, et al. Liver transplantation and aortic valve replacement. Int J Organ 
Transplant Med. 2011;2(1):32-36.

6. Lima B, Nowicki ER, Miller CM, Hashimoto K, Smedira NG, Gonzalez-Stawinski GV. Outcomes of simultaneous liver transplantation and elective cardiac surgical procedures. Ann Thorac Surg. 2011;92(5):1580-1584.

7. Harrison JD, Selzman CH, Thiesset HF, Box T, Hutson WR, Lu JK, Campsen J, et al. Minimally invasive aortic valve replacement with orthotopic liver transplantation: report of a case. Surg Today. 2014;44(3):546-549.
8. Gelsomino S, Morocutti G, Masullo G, Cheli G, Poldini F, Da Broi U, Livi U. Open heart surgery in patients with dialysis-dependent renal insufficiency. J Card Surg. 2001;16(5):400-407.

9. Tekin S, Zengin M, Tekin I, Yucetin L, Yavuz HA, Okutan H, Demirbas A. Simultaneous Cardiac Surgery and Renal Transplantation Compared With Renal Transplantation After Cardiac Surgery. Transplant Proc. 2015;47(5):13401344. 